\title{
Rational Bundles and Recursion Operators for Integrable Equations on A.III-type Symmetric Spaces
}

\author{
V. S. Gerdjikov ${ }^{1}$, G. G. Grahovski1 ${ }^{1,2}$, A. V. Mikhailov ${ }^{3}$, \\ T. I. Valchev ${ }^{1}$ \\ ${ }^{1}$ Institute of Nuclear Research and Nuclear Energy, Bulgarian Academy of Sciences, 72 \\ Tsarigradsko chausee, Sofia 1784, Bulgaria \\ ${ }^{2}$ School of Mathematical Sciences, Dublin Institute of Technology, \\ Kevin Street, Dublin 8, Ireland \\ ${ }^{3}$ Applied Math. Department, University of Leeds, \\ Woodhouse Lane, Leeds, LS2 9JT, UK \\ E-mails: gerjikov@inrne.bas.bg, grah@inrne.bas.bg \\ a.v.mikhailov@leeds.ac.uk, valtchev@inrne.bas.bg
}

\begin{abstract}
We analyze and compare the methods of construction of the recursion operators for a special class of integrable nonlinear differential equations related to A.III-type symmetric spaces in Cartan's classification and having additional reductions.
\end{abstract}

Key words: Rational bundle, Integrable equations, Recursion operators

\section{Introduction}

Recursion operators $\Lambda_{ \pm}$play an important role in the theory of the nonlinear evolution equations (NLEE), integrable by the inverse scattering method. They have been constructed and analyzed for a wide class of Lax operators $L$ and appeared to generate not only the Lax representations, but also the hierarchy of NLEE's related to a given Lax operator $L$, their conservation laws and their hierarchy of Hamiltonian structures, see $[3,24,22,8]$ and the numerous references therein. Such operators can be viewed also as the Lax $L$ operator, taken in the adjoint representation of the relevant Lie algebra $\mathfrak{g}$.

The construction of $\Lambda_{ \pm}$for Lax operators, whose explicit dependence on the spectral parameter $\lambda$ is comparatively simple (say, linear, or quadratic) has been done a long time ago [1, 12, 4, 5. Furthermore, the completeness property for the set of eigenfunctions of $\Lambda_{ \pm}$(the 'squared solutions' of $L$ ) is of paramount importance. The completeness of the 'squared solutions' plays a fundamental role in proving that the ISM is, in fact, a nonlinear analogue of the Fourier transform, which allows one to linearize the NLEE. Using these relations one is able to derive all fundamental properties of the NLEE on a common basis.

An important tool of extracting new NLEE from a known multi-component ones, is the the reduction group, introduced in [14, 15, 16]. It led to the discovery of the 2-dimensional Toda field theories [16, 20]. Latest developments of the method led to the discovery of new automorphic Lie algebras and their classification [13, 18, 19].

The problem of deriving recursion operators becomes more difficult when we impose additional reductions on $L$. If this additional reduction is compatible with $L$, being linear or quadratic in $\lambda$, the construction of $\Lambda$ is not a difficult task, see [8]. An alternative construction of $\Lambda$ as formal recursion operator is given in [10, 9], see also the review papers [2, 21]. The effect of the $\mathbb{Z}_{n}$-reduction is as follows: i) the relevant 'squared solutions' have analyticity properties in sectors of the complex $\lambda$-plane closing angles $\pi / n$; ii) the grading 
of the Lie algebra $\mathfrak{g} \equiv \bigoplus_{k=0}^{n-1} \mathfrak{g}^{(k)}$ is more involved and as a consequence the recursion operator is factorized into a product of $n$ factors $\Lambda=\prod_{k=0}^{n-1} \Lambda_{k}$, and each of the factors $\Lambda_{k}$ maps $\Lambda_{k}: \mathfrak{g}^{(k-1)} \rightarrow \mathfrak{g}^{(k)}$.

The situation becomes more complicated if the additional reduction drastically changes the $\lambda$-dependence of the Lax operator. Here we address this problem for one of the simplest nontrivial cases when the Lax operator $L$, due to an additional $\mathbb{Z}_{2}$-reduction changes its $\lambda$-dependence from polynomial into rational one. The linear in $\lambda$ Lax operator

$$
L_{0} \psi \equiv i \partial_{x} \psi+\lambda L_{1} \psi(x, \lambda)=0,
$$

was shown to give rise [10, 6] to the integrable system

$$
i \boldsymbol{u}_{t}=\left(\left(1-\boldsymbol{u} \boldsymbol{u}^{\dagger}\right) \boldsymbol{u}_{x}\right)_{x}, \quad \boldsymbol{u}^{\dagger} \boldsymbol{u}=\mathbb{1}
$$

where $\boldsymbol{u}$ is $(N-k) \times k$ complex matrix and $\mathbb{1}$ is the unit matrix. The system (1.2) is $S(U(N-k) \times U(k))$ invariant, and, in this sense, is isotropic. In particular, if $k=1$, equation (1.2) can be seen as a $U(N-1)$ invariant integrable system on $\mathbb{C P}^{N-1}$. The corresponding recursion operators, relevant for the fundamental properties of eq. (1.2), were derived in [7].

In [6] we derived new NLEE with additional $\mathbb{Z}_{2}$-reduction, which maps $\lambda \rightarrow \lambda^{-1}$. This reduction is among the simplest nontrivial ones, see [14, 15, 16, 18, 19]. Due to it, the relevant Lax operator acquires a rational dependence of $\lambda$ :

$$
L \psi \equiv i \partial_{x} \psi+\left(\lambda L_{1}+\frac{1}{\lambda} L_{-1}\right) \psi=0
$$

and the simplest NLEE take the form

$$
\begin{aligned}
i u_{t} & =u_{x x}-\left(u\left(u^{*} u_{x}+v^{*} v_{x}\right)\right)_{x}+8 v v^{*} u \\
i v_{t} & =v_{x x}-\left(v\left(u^{*} u_{x}+v^{*} v_{x}\right)\right)_{x}-8 u u^{*} v
\end{aligned}
$$

where $u$ and $v$ are functions of $x$ and $t$ subject to the condition:

$$
\left|u^{2}\right|+\left|v^{2}\right|=1
$$

i.e. the vector with components $u$ and $v$ sweeps a 3 -dimensional sphere in $\mathbb{R}^{4}$. System (1.4) can also be seen as an anisotropic deformation of (1.2) with $k=1, N=3$.

Our main aim in the present paper is to present two ways of deriving the recursion operators related to the Lax operators with rational dependence on $\lambda$. It is a natural extensions of our previous results [6, 7]. In the next Section 2 we give preliminaries concerning the spectral properties of $L$ (1.3). In Section 3 we derive the recursion operator using the Gürses-Karasu-Sokolov (GKS) method. In Section 4 we use the Wronskian relations to determine the "squared solutions of $L$. Next using the gauge covariant approach [1, 8] we introduce the recursion operator as the one for which the 'squared solutions' are eigenfunctions. In the last Section 5 we give some conclusions, while the Appendix contains some details from our calculations.

\section{Preliminaries}

In this section we first formulate the basic results from our previous paper [6]. There we have shown that eq. (1.4), which is naturally related to the symmetric space [11] of 
A.III-type $S U(3) / S(U(1) \times U(2))$, allows a Lax representation and can be solved by the inverse scattering method. The Lax operator $L$ is given by eq. (1.3) and the equation itself is the compatibility condition between $L$ and the linear operator

$$
A \psi:=i \partial_{t} \psi+\left(A_{0}+\lambda A_{1}+\frac{1}{\lambda} A_{-1}+\lambda^{2} A_{2}+\frac{1}{\lambda^{2}} A_{-2}\right) \psi=\psi f(\lambda),
$$

It is well know the above mentioned symmetric space is constructed by using Cartan's involutive automorphism [11], which induces a $\mathbb{Z}_{2}$ grading in the underlying Lie algebra

$$
\begin{aligned}
\mathfrak{g} & =\mathfrak{g}^{(\mathbf{0})} \oplus \mathfrak{g}^{(\mathbf{1})}, \quad J_{1}=\operatorname{diag}(1,-1,-1) \\
\mathfrak{g}^{(0)} & =\left\{Y \in \mathfrak{g}: J_{1} Y J_{1}=Y\right\}, \quad \mathfrak{g}^{(1)}=\left\{X \in \mathfrak{g}: J_{1} X J_{1}=-X\right\} .
\end{aligned}
$$

Our Lax representation is such that $L_{ \pm 1}, A_{ \pm 1} \in \mathfrak{g}^{1}$ and $A_{0}, A_{ \pm 2} \in \mathfrak{g}^{0}$, for more details see the Appendix. In particular, for $L_{1}$ and $A_{1}$ we write

$$
L_{1}=\left(\begin{array}{ccc}
0 & u & v \\
u^{*} & 0 & 0 \\
v^{*} & 0 & 0
\end{array}\right), \quad A_{1}=\left(\begin{array}{ccc}
0 & a & b \\
a^{*} & 0 & 0 \\
b^{*} & 0 & 0
\end{array}\right)
$$

which explicitly involves the first two reduction conditions:

$$
L_{ \pm 1}^{\dagger}=L_{ \pm 1}, \quad A_{ \pm 1}^{\dagger}=A_{ \pm 1}, \quad A_{ \pm 2}^{\dagger}=A_{ \pm 2} .
$$

We impose also a third reduction of the form

$$
J_{2} L_{1} J_{2}=L_{-1}, \quad J_{2} A_{1} J_{2}=A_{-1}, \quad J_{2} A_{2} J_{2}=A_{-2}
$$

where $J_{2}=\operatorname{diag}(1,-1,1)$. At this point, we impose one additional requirement

$$
|u|^{2}+|v|^{2}=1
$$

that is the vector $(u, v)$ lives in a 3 -dimensional sphere in $\mathbb{R}^{4}$.

The spectral theory of the Lax operator $L(1.3)$ substantially depends on the boundary conditions. We have two natural ways to fix the boundary configurations, namely:

1. $\lim _{x \rightarrow \pm \infty} u(x, t)=1, \quad \lim _{x \rightarrow \pm \infty} v(x, t)=0$

2. $\lim _{x \rightarrow \pm \infty} u(x, t)=0, \quad \lim _{x \rightarrow \pm \infty} v(x, t)=1$

The Jost solutions of $L$ are determined by using the asymptotic potentials $U_{ \pm, a s}(\lambda)=$ $\lim _{x \rightarrow \pm \infty}\left(\lambda L_{1}+\lambda^{-1} L_{-1}\right)$ which can be diagonalised for both choices of boundary conditions

$$
\begin{aligned}
g_{0}^{-1} U_{ \pm, \mathrm{as}}(\lambda) g_{0} & =J_{\mathrm{a}}(\lambda), \quad g_{0}=\frac{1}{\sqrt{2}}\left(\begin{array}{ccc}
1 & 0 & 1 \\
1 & 0 & -1 \\
0 & -\sqrt{2} & 0
\end{array}\right) \\
J_{\mathrm{a}}(\lambda) & =\left(\lambda-\lambda^{-1}\right) \operatorname{diag}(1,0,-1) \\
g_{0}^{-1} U_{ \pm, \mathrm{as}}(\lambda) g_{0} & =J_{\mathrm{b}}(\lambda), \quad g_{0}=\frac{1}{\sqrt{2}}\left(\begin{array}{ccc}
1 & 0 & -1 \\
0 & \sqrt{2} & 0 \\
1 & 0 & 1
\end{array}\right) \\
J_{\mathrm{b}}(\lambda) & =\left(\lambda+\lambda^{-1}\right) \operatorname{diag}(1,0,-1) .
\end{aligned}
$$




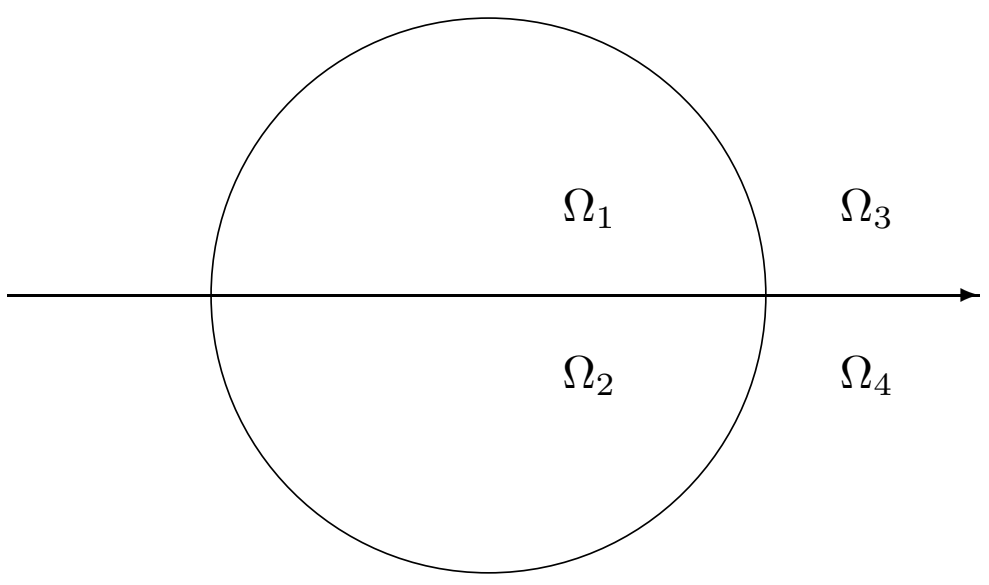

Figure 1: Continuous spectrum of $L$, case b).

The continuous part of the spectrum of $L$ for the case a) fills in the real axis $\mathbb{R}$ while for the case b) it is the union of the real axis and the unit circle, see Fig 1.

In [6] we have constructed the fundamental analytic solutions (FAS) of $L$ for both cases. Skipping the details, we note only that these FAS can be viewed as solutions of Riemann-Hilbert problems (RHP):

The Jost solutions of $L$ and the related scattering matrix $T(\lambda)$ are defined as follows:

$$
\begin{aligned}
\lim _{x \rightarrow \pm \infty} \psi_{ \pm}(x, \lambda) e^{-i J(\lambda) x} g_{0}^{-1} & =\mathbb{1}, \\
T(\lambda) & =\psi_{+}^{-1}(x, \lambda) \psi_{-}(x, \lambda)
\end{aligned}
$$

For the case a) the fundamental analytic solutions $\chi^{+}$and $\chi^{-}$are solutions of the following RHP

$$
\chi^{+}(x, \lambda)=\chi^{-}(x, \lambda) G(\lambda), \quad G(\lambda)=\left[S^{-}(\lambda)\right]^{-1} S^{+}(\lambda), \quad \lambda \in \mathbb{R} .
$$

They obey the symmetry relations imposed by the reductions

$$
\begin{aligned}
\left(\chi^{+}\right)^{\dagger}\left(x, \lambda^{*}\right) & =\chi^{-}(x, \lambda) \\
J_{1} \chi^{+}(x,-\lambda) J_{1} & =\chi^{-}(x, \lambda) \\
J_{2} \chi^{ \pm}(x, 1 / \lambda) J_{2} & =\chi^{ \pm}(x, \lambda) .
\end{aligned}
$$

In the case b) we have 4 fundamental analytic solutions $\chi^{(j)}$ with analyticity regions $\Omega_{j}$, which satisfy a RHP on the contour shown on Figure 1:

$$
\begin{array}{ll}
\chi^{(1)}(x, \lambda)=\chi^{(2)}(x, \lambda) G(\lambda), & \lambda \in[-1,1] \\
\chi^{(4)}(x, \lambda)=\chi^{(3)}(x, \lambda) G(\lambda), & \lambda \in(-\infty,-1] \cup[1, \infty) \\
\chi^{(1)}(x, \lambda)=\chi^{(3)}(x, \lambda) G(\lambda), & \lambda=e^{i \varphi}, \quad \varphi \in(0, \pi) \\
\chi^{(4)}(x, \lambda)=\chi^{(2)}(x, \lambda) G(\lambda), & \lambda=e^{i \varphi}, \quad \varphi \in(\pi, 2 \pi)
\end{array}
$$

where the sewing function is given by

$$
G(\lambda)=\left(S^{-}(\lambda)\right)^{-1} S^{+}(\lambda)
$$

The FAS obey the following symmetry relations:

$$
\begin{aligned}
& {\left[\chi^{(1)}\left(x, \lambda^{*}\right)\right]^{\dagger}=\left[\chi^{(2)}(x, \lambda)\right]^{-1}, \quad\left[\chi^{(4)}\left(x, \lambda^{*}\right)\right]^{\dagger}=\left[\chi^{(3)}(x, \lambda)\right]^{-1}} \\
& J_{1} \chi^{(1)}(x,-\lambda) J_{1}=\chi^{(2)}(x, \lambda), \quad J_{1} \chi^{(4)}(x,-\lambda) J_{1}=\chi^{(3)}(x, \lambda) \\
& J_{2} \chi^{(1)}(x, 1 / \lambda) J_{2}=\chi^{(4)}(x, \lambda), \quad J_{2} \chi^{(2)}(x, 1 / \lambda) J_{2}=\chi^{(3)}(x, \lambda) \text {. }
\end{aligned}
$$




\section{Recursion operators}

This section is dedicated to the construction of recursion operator for the NLEE (1.4). For this to be done we are applying the method proposed by Gürses, Karasu and Sokolov (GKS) [10]. The recursion operator is viewed here a pseudo-differential operator to map a symmetry of a NLEE into another symmetry. The underlying idea of the GKS method is to interrelate two adjacent flows $\check{A}$ and $A$ with evolution variables $\tau$ and $t$ respectively through a equality in the form [10, 23]

$$
\check{V}=\left(\lambda^{2}+\lambda^{-2}\right) V+B .
$$

The matrix-valued functions $\tilde{V}$ and $V$ are involved in the Lax operators $\tilde{A}$ and $A$ as follows

$$
\begin{aligned}
& \tilde{A}=i \partial_{\tau}+\tilde{V}(x, t, \lambda), \\
& A=i \partial_{t}+V(x, t, \lambda)
\end{aligned}
$$

All quantities above must be invariant under the action of the $\mathbb{Z}_{2}$ reductions (2.4) and (2.5). This explains the choice of the coefficient before $V$ in (3.1) which is invariant under $\lambda \rightarrow-\lambda$ and $\lambda \rightarrow \lambda^{-1}$. The operator $B$ is chosen to be a rational function of $\lambda$

$$
B=B_{0}+\lambda B_{1}+\frac{1}{\lambda} B_{-1}+\lambda^{2} B_{2}+\frac{1}{\lambda^{2}} B_{-2}, \quad B_{-k}=J_{2} B_{k} J_{2}, \quad k=1,2
$$

The matrices involved in $B$ are hermitian ones and have a block structure given by

$$
B_{1} \equiv\left(\begin{array}{cc}
0 & \mathbf{c}^{T} \\
\mathbf{c}^{*} & 0
\end{array}\right), \quad B_{2} \equiv\left(\begin{array}{cc}
d & 0 \\
0 & D
\end{array}\right), \quad D \equiv\left(\begin{array}{cc}
\alpha & \beta \\
\beta^{*} & \delta
\end{array}\right)
$$

in a complete analogy with the second Lax operator (2.1).

At the begining of our consideration, let us remark that the zero curvature condition of the Lax operators $L$ and $A$ could be written in the following manner:

$$
i L_{t} \equiv[L, V]
$$

where $L_{t} \equiv \lambda L_{1, t}+\lambda^{-1} L_{-1, t}$. After substituting (3.1) in the analog of (3.6), when the evolution paramenter $t$ is replaced by $\tau$, we obtain the following basic equation:

$$
i L_{\tau}=i\left(\lambda^{2}+\lambda^{-2}\right) L_{t}+[L, B] .
$$

Then the recursion operator $\mathcal{R}$ acts as follows:

$$
\left(\begin{array}{c}
\mathbf{u} \\
\mathbf{u}^{*}
\end{array}\right)_{\tau}=\mathcal{R}\left(\begin{array}{c}
\mathbf{u} \\
\mathbf{u}^{*}
\end{array}\right)_{t}
$$

Since all matrices are traceless, we have

$$
d=-\operatorname{tr} D=-(\alpha+\delta) .
$$

By comparing coefficients before different powers of $\lambda$, one splits equation (3.7) into

$$
i L_{1, t}+\left[L_{1}, B_{2}\right]=0 \quad \Rightarrow \quad i \mathbf{u}_{t}+\left(D^{*}-d\right) \mathbf{u}=0
$$




$$
\begin{aligned}
& i B_{2, x}+\left[L_{1}, B_{1}\right]=0 \Rightarrow \begin{array}{l}
i D_{x}+\mathbf{u}^{*} \mathbf{c}^{T}-\mathbf{c}^{*} \mathbf{u}^{T}=0 \\
i d_{x}+\mathbf{u}^{T} \mathbf{c}^{*}-\mathbf{c}^{T} \mathbf{u}^{*}=0
\end{array} \\
& i L_{1, \tau}=i L_{-1, t}+i B_{1, x}+\left[L_{1}, B_{0}\right]+\left[L_{-1}, B_{2}\right] \\
& i B_{0, x}+\left[L_{1}, B_{-1}\right]+\left[L_{-1}, B_{1}\right]=0 .
\end{aligned}
$$

The rest of relations could be obtained from those above, by multiplying each quantity by $J_{2}$ on its left and on its right hand side.

The system (3.9) is linear for the matrix elements of $D$. A solution to (3.9) is given by

$$
\begin{aligned}
\alpha & =i\left(u u_{t}^{*}+v^{*} v_{t}\right)-d\left(2|v|^{2}-|u|^{2}\right) \quad \beta=i\left(v u_{t}^{*}-u^{*} v_{t}\right)+3 d u^{*} v \\
\delta & =-i\left(u u_{t}^{*}+v^{*} v_{t}\right)-d\left(2|u|^{2}-|v|^{2}\right) .
\end{aligned}
$$

The solvability condition for the system of linear equations (3.13) is:

$$
\alpha_{x}|v|^{2}+\delta_{x}|u|^{2}-\beta_{x} u v^{*}-\beta_{x}^{*} u^{*} v=0
$$

One can check that

$$
\mathbf{c}=\left(\begin{array}{c}
c \\
s
\end{array}\right)=\frac{i}{2}\left(\begin{array}{c}
u\left(\delta_{x}-\alpha_{x}\right)-2 v \beta_{x}^{*} \\
-v\left(\delta_{x}-\alpha_{x}\right)-2 u \beta_{x}
\end{array}\right)+\kappa\left(\begin{array}{l}
u \\
v
\end{array}\right)
$$

where $\kappa$ is real, but otherwise arbitrary, is the general solution of (3.13). In what follows we shall set $\kappa=0$ without any loss of generality. It could be written in the following matrix form

$$
\left(\begin{array}{c}
c \\
s \\
c^{*} \\
s^{*}
\end{array}\right)_{x}=\mathcal{A}\left(\begin{array}{c}
\beta \\
\beta^{*} \\
\frac{\alpha-\delta}{2} \\
\frac{\alpha+\delta}{2}
\end{array}\right), \quad \mathcal{A}:=i \frac{\mathrm{d}}{d x}\left(\begin{array}{cccc}
0 & -v & -u & 0 \\
-u & 0 & v & 0 \\
v^{*} & 0 & u^{*} & 0 \\
0 & u^{*} & -v^{*} & 0
\end{array}\right) \frac{\mathrm{d}}{d x}
$$

Next step is to find the function $d$, involved in the expressions for $\alpha, \beta$ etc. For this to be done, we make use of the condition (3.14) which leads to a linear differential equation for $d$ :

$$
\begin{aligned}
-2 d_{x} & +\mathrm{i}\left(u^{*} u_{t}+v^{*} v_{t}\right)_{x}-i\left[\left(u u_{t}^{*}+v^{*} v_{t}\right)\left(|v|^{2}-|u|^{2}\right)_{x}\right. \\
& \left.-\left(u v_{t}^{*}-v^{*} u_{t}\right)\left(u^{*} v\right)_{x}+\left(u^{*} v_{t}-v^{*} u_{t}\right)\left(u v^{*}\right)_{x}\right]=0 .
\end{aligned}
$$

After some simple manipulations one obtains

$$
d=-\frac{i}{2}\left(u u_{t}^{*}+v v_{t}^{*}\right)-\frac{i}{2} \partial_{x}^{-1}\left(u_{t} u_{x}^{*}+v_{t} v_{x}^{*}-u_{t}^{*} u_{x}-v_{t}^{*} v_{x}\right)
$$

where $\partial_{x}^{-1}:=\int_{ \pm \infty}^{x} d y$.

To calculate $\mathcal{R}$, one needs the diagonal matrix $B_{0}$. It directly follows from (3.12) that the following equation holds true

$$
i B_{0, x}=2\left(\begin{array}{ccc}
u c^{*}-u^{*} c+v^{*} s-v s^{*} & 0 & 0 \\
0 & u^{*} c-u c^{*} & 0 \\
0 & 0 & v s^{*}-v^{*} s
\end{array}\right) .
$$


Taking into account formulas (3.10), one deduces that

$$
B_{0}=2\left(\begin{array}{ccc}
\alpha-\delta & 0 & 0 \\
0 & -\alpha & 0 \\
0 & 0 & \delta
\end{array}\right)
$$

Furthermore, taking into account the structure of (3.11), one can easily see that $\mathcal{R}$ can be split in the following form:

$$
\mathcal{R}=\mathcal{C}+\mathcal{R}_{0}+\mathcal{D}
$$

The first term $\mathcal{C}$ originates from the first term in (3.11), and it is a constant matrix $\operatorname{diag}(-1,1,-1,1)$. The third operator $\mathcal{D}$ is obtained from the two commutators in (3.11). It turns out that it splits into a local term

$$
\mathcal{D}_{\text {loc }}:=\left(\begin{array}{cccc}
8|u|^{2}-1 & -4 u^{2}\left(3|v|^{2}-1\right) & 0 & 12 u v|u|^{2} \\
0 & -12 u v|v|^{2} & 1-8|v|^{2} & 4 v^{2}\left(3|u|^{2}-1\right) \\
-4\left(u^{*}\right)^{2}\left(3|v|^{2}-1\right) & 8|u|^{2}-1 & 12 u^{*} v^{*}|u|^{2} & 0 \\
-12 u^{*} v^{*}|v|^{2} & 0 & 4\left(v^{*}\right)^{2}\left(3|u|^{2}-1\right) & 1-8|v|^{2}
\end{array}\right)
$$

and a nonlocal one

$$
\mathcal{D}_{\text {nonl }}=4\left(\begin{array}{c}
u\left(1-3|v|^{2}\right) \\
v\left(3|u|^{2}-1\right) \\
u^{*}\left(3|v|^{2}-1\right) \\
v^{*}\left(1-3|u|^{2}\right)
\end{array}\right) \partial_{x}^{-1}\left[\left(u_{x}^{*}, v_{x}^{*},-u_{x},-v_{x}\right) \cdot\right]
$$

Finally the operator $\mathcal{R}_{0}=\mathcal{A}\left(\mathcal{B}_{\text {loc }}+\mathcal{B}_{\text {nonl }}\right)$ splits into a local and a nonlocal parts as follows:

$$
\begin{aligned}
\mathcal{B}_{\text {loc }} & =\frac{i}{4}\left(\begin{array}{cccc}
0 & -4 u^{*} & 2 v\left(3|v|^{2}-1\right) & -6 u^{*} v^{2} \\
2 v^{*}\left(1-3|v|^{2}\right) & 6 u\left(v^{*}\right)^{2} & 0 & 4 u \\
-4 u^{*} & 0 & 3 u\left(|v|^{2}-|u|^{2}\right) & -v\left(1+6|u|^{2}\right) \\
-u^{*} & -v^{*} & 0 & 0
\end{array}\right) \\
\mathcal{B}_{\text {nonl }} & =-\frac{3 i}{4}\left(\begin{array}{c}
2 u^{*} v \\
2 u v^{*} \\
|u|^{2}-|v|^{2} \\
-1 / 3
\end{array}\right) \partial_{x}^{-1}\left[\left(u_{x}^{*}, v_{x}^{*},-u_{x},-v_{x}\right)\right]
\end{aligned}
$$

In fact, $\mathcal{R}_{0}$ represents the recursion operator in the case of polynomial bundle Lax pair [7]:

$$
\begin{aligned}
& L:=i \partial_{x}+\lambda L_{1}, \\
& A:=i \partial_{t}+\lambda A_{1}+\lambda^{2} A_{2}
\end{aligned}
$$

\section{Recursion operators and 'squared solutions'}

This approach is based on the Wronskian relations, mapping the potential of the Lax operator $L$ onto the scattering data, which allow one to introduce the 'squared solutions' for $L$ (see, e.g. [8] and the references therein). 
The Wronskian relations, derived in [6], take the form:

$$
\begin{array}{r}
\left.\left\langle i \chi^{-1} J_{0} \chi(x, \lambda)-i J_{0}, E_{\alpha}\right\rangle\right|_{x=-\infty} ^{\infty}=\int_{x=-\infty}^{\infty} d x\left\langle\left[L_{1}, J_{0}\right], \Phi_{1}(x, \lambda)\right\rangle \\
\left.i\left\langle\chi^{-1} \delta \chi(x, \lambda), E_{\alpha}\right\rangle\right|_{x=-\infty} ^{\infty}=-\int_{x=-\infty}^{\infty} d x\left\langle\delta L_{1}, \Phi_{1}(x, \lambda)\right\rangle
\end{array}
$$

where we have introduced the squared solutions:

$$
\Phi_{1}(x, \lambda)=\lambda e_{\alpha}(x, \lambda)+\lambda^{-1} \varphi_{0}\left(e_{\alpha}\right)(x, \lambda)
$$

and

$$
e_{\alpha}(x, \lambda)=\chi E_{\alpha} \chi^{-1}(x, \lambda) .
$$

where $E_{\alpha}$ is one of the Cartan-Weyl generators.

In this approach, see [1, 8], one picks up a certain 'projections' of the 'squared solutions' (see $K_{1 ; \alpha}^{ \pm, \perp}(x, \lambda)$ in eq. (4.12) below) which contribute to the right hand sides of eq. (4.1). Then the recursion operators can be introduced as the ones, that have $K_{1 ; \alpha}^{ \pm, \perp}(x, \lambda)$ as eigenfunctions.

Let us introduce

$$
J_{1}=\operatorname{diag}(1,-1,-1), \quad K_{1}=\operatorname{diag}(1,0,-1), \quad K_{0}=\operatorname{diag}(1,-1,1)
$$

and

$$
\Phi_{k ; \alpha}^{ \pm}(x, \lambda)=\lambda^{k} e_{\alpha}^{ \pm}(x, \lambda)+\lambda^{-k} \varphi_{0}\left(e_{\alpha}^{ \pm}(x, \lambda)\right),
$$

which satisfy the equations:

$$
\begin{gathered}
i \frac{\partial \Phi_{k ; \alpha}}{\partial x}+\left[L_{1}, \Phi_{k+1 ; \alpha}^{ \pm}(x, \lambda)\right]+\left[L_{-1}, \Phi_{k-1 ; \alpha}^{ \pm}(x, \lambda)\right]=0 \\
\varphi_{0}\left(\Phi_{k ; \alpha}^{ \pm}(x, \lambda)\right)=\Phi_{-k ; \alpha}^{ \pm}(x, \lambda), \quad \varphi_{0}\left(\Phi_{0 ; \alpha}^{ \pm}(x, \lambda)\right)=\Phi_{0 ; \alpha}^{ \pm}(x, \lambda) \\
i \frac{\partial \Phi_{0 ; \alpha}}{\partial x}+\left(\mathbb{1}+\varphi_{0}\right)\left[L_{1}, \Phi_{1 ; \alpha}^{ \pm}(x, \lambda)\right]=0
\end{gathered}
$$

In addition, we have:

$$
\left(\lambda+\lambda^{-1}\right) \Phi_{k ; \alpha}^{ \pm}(x, \lambda)=\Phi_{k+1 ; \alpha}^{ \pm}(x, \lambda)+\Phi_{k-1 ; \alpha}^{ \pm}(x, \lambda)
$$

In what follows we will use only the equations for $\Phi_{0 ; \alpha}^{ \pm}(x, \lambda)$ and $\Phi_{1 ; \alpha}^{ \pm}(x, \lambda)$ :

$$
\begin{aligned}
i \frac{\partial \Phi_{0 ; \alpha}}{\partial x}+\left(\mathbb{1}+\varphi_{0}\right)\left[L_{1}, \Phi_{1 ; \alpha}^{ \pm}(x, \lambda)\right] & =0 \\
i \frac{\partial \Phi_{1 ; \alpha}}{\partial x}-\left[L_{1}-L_{-1}, \Phi_{0 ; \alpha}^{ \pm}(x, \lambda)\right] & =-\left(\lambda+\lambda^{-1}\right)\left[L_{1}, \Phi_{1 ; \alpha}^{ \pm}(x, \lambda)\right]
\end{aligned}
$$

Next, we insert the splitting:

$$
\begin{aligned}
\Phi_{0 ; \alpha}^{ \pm}(x, \lambda) & =H_{0 ; \alpha}^{ \pm}(x, \lambda)+K_{0 ; \alpha}^{ \pm}(x, \lambda), & \Phi_{1 ; \alpha}^{ \pm}(x, \lambda) & =H_{1 ; \alpha}^{ \pm}(x, \lambda)+K_{1 ; \alpha}^{ \pm}(x, \lambda) \\
H_{0 ; \alpha}^{ \pm}(x, \lambda) & =\varphi_{0}\left(H_{0 ; \alpha}^{ \pm}(x, \lambda)\right), & K_{0 ; \alpha}^{ \pm}(x, \lambda) & =\varphi_{0}\left(K_{0 ; \alpha}^{ \pm}(x, \lambda)\right)
\end{aligned}
$$


and obtain

$$
\begin{aligned}
& i \frac{\partial H_{0 ; \alpha}^{ \pm}}{\partial x}+\left(\mathbb{1}+\varphi_{0}\right)\left[L_{1}, K_{1 ; \alpha}^{ \pm}(x, \lambda)\right]=0 \\
& i \frac{\partial K_{0 ; \alpha}^{ \pm}}{\partial x}+\left(\mathbb{1}+\varphi_{0}\right)\left[L_{1}, H_{1 ; \alpha}^{ \pm}(x, \lambda)\right]=0 \\
& i \frac{\partial H_{1 ; \alpha}^{ \pm}}{\partial x}-\left[L_{1}-L_{-1}, K_{0 ; \alpha}^{ \pm}(x, \lambda)\right]=-\left(\lambda+\lambda^{-1}\right)\left[L_{1}, K_{1 ; \alpha}^{ \pm}(x, \lambda)\right] \\
& i \frac{\partial K_{1 ; \alpha}^{ \pm}}{\partial x}-\left[L_{1}-L_{-1}, H_{0 ; \alpha}^{ \pm}(x, \lambda)\right]=-\left(\lambda+\lambda^{-1}\right)\left[L_{1}, H_{1 ; \alpha}^{ \pm}(x, \lambda)\right]
\end{aligned}
$$

The integration the first two of the above equations gives:

$$
\begin{aligned}
& H_{0 ; \alpha}^{ \pm}(x, \lambda)=h_{00 ; \alpha}^{ \pm}+i\left(\mathbb{1}+\varphi_{0}\right) \partial_{x}^{-1}\left[L_{1}, K_{1 ; \alpha}^{ \pm, \perp}(x, \lambda)\right] \\
& K_{0 ; \alpha}^{ \pm}(x, \lambda)=k_{00 ; \alpha}^{ \pm}+i\left(\mathbb{1}+\varphi_{0}\right) \partial_{x}^{-1}\left[L_{1}, H_{1 ; \alpha}^{ \pm, \perp}(x, \lambda)\right],
\end{aligned}
$$

where $h_{00 ; \alpha}^{ \pm}$and $k_{00 ; \alpha}^{ \pm}$are matrix-valued constants. Thus we expressed $K_{0 ; \alpha}^{ \pm}$and $H_{0 ; \alpha}^{ \pm}$in terms of $K_{1 ; \alpha}^{ \pm, \perp}$ and $H_{1 ; \alpha}^{ \pm, \perp}$ where

$$
\begin{aligned}
& H_{1 ; \alpha}^{ \pm, \perp}(x, \lambda)=H_{1 ; \alpha}^{ \pm}(x, \lambda)-\frac{3}{2} L_{2}\left\langle H_{1 ; \alpha}^{ \pm}, L_{2}\right\rangle \\
& K_{1 ; \alpha}^{ \pm, \perp}(x, \lambda)=K_{1 ; \alpha}^{ \pm}(x, \lambda)-\frac{1}{2} L_{1}\left\langle K_{1 ; \alpha}^{ \pm}, L_{1}\right\rangle
\end{aligned}
$$

where $L_{2}=L_{1}^{2}-2 / 31$. Let us now derive the equations for $K_{1 ; \alpha}^{ \pm, \perp}$ and $H_{1 ; \alpha}^{ \pm, \perp}$. To do this, we insert eq. (4.12 and insert it into the last two equations of (4.10). Thus we get:

$$
\begin{aligned}
i \frac{\partial H_{1 ; \alpha}^{ \pm, \perp}}{\partial x} & +\frac{3 i}{2} L_{2, x}\left\langle H_{1 ; \alpha}^{ \pm}, L_{2}\right\rangle+\frac{3 i}{2} L_{2} \frac{\partial\left\langle H_{1 ; \alpha}^{ \pm}, L_{2}\right\rangle}{\partial x}-\left[L_{1}-L_{-1}, K_{0 ; \alpha}^{ \pm}(x, \lambda)\right] \\
& =-\left(\lambda+\lambda^{-1}\right)\left[L_{1}, K_{1 ; \alpha}^{ \pm}(x, \lambda)\right] \\
i \frac{\partial K_{1 ; \alpha}^{ \pm, \perp}}{\partial x} & +\frac{i}{2} L_{1, x}\left\langle K_{1 ; \alpha}^{ \pm}, L_{1}\right\rangle+\frac{i}{2} L_{1} \frac{\partial\left\langle K_{1 ; \alpha}^{ \pm}, L_{1}\right\rangle}{\partial x}-\left[L_{1}-L_{-1}, H_{0 ; \alpha}^{ \pm}(x, \lambda)\right] \\
& =-\left(\lambda+\lambda^{-1}\right)\left[L_{1}, H_{1 ; \alpha}^{ \pm}(x, \lambda)\right] .
\end{aligned}
$$

Next, we separate the left hand sides of eq. (4.13) into terms commuting with $L_{1}$ and $L_{2}$ and terms 'orthogonal' to them. In order to calculate the coefficients $\left\langle H_{1: \alpha}^{ \pm}, L_{2}\right\rangle$ and $\left\langle K_{1 ; \alpha}^{ \pm}, L_{1}\right\rangle$, we multiply both sides of the first (resp. the second) of the eqs. (4.13) by $L_{2}$ (resp. by $L_{1}$ ) and take the trace. The result is:

$$
\begin{aligned}
& i\left\langle\frac{\partial H_{1 ; \alpha}^{ \pm, \perp}}{\partial x}, L_{2}\right\rangle+i \frac{\partial\left\langle H_{1 ; \alpha}^{ \pm}, L_{2}\right\rangle}{\partial x}-\left\langle\left[L_{1}-L_{-1}, K_{0 ; \alpha}^{ \pm}(x, \lambda)\right], L_{2}\right\rangle=0 \\
& i\left\langle\frac{\partial K_{1 ; \alpha}^{ \pm, \perp}}{\partial x}, L_{1}\right\rangle+i \frac{\partial\left\langle K_{1 ; \alpha}^{ \pm}, L_{1}\right\rangle}{\partial x}-\left\langle\left[L_{1}-L_{-1}, H_{0 ; \alpha}^{ \pm}(x, \lambda)\right], L_{1}\right\rangle=0
\end{aligned}
$$

Integrating (4.14) we get:

$$
\begin{aligned}
& \left\langle H_{1 ; \alpha}^{ \pm}, L_{2}\right\rangle=h_{01, \alpha}^{ \pm}-\partial_{x}^{-1}\left\langle\frac{\partial H_{1 ; \alpha}^{ \pm, \perp}}{\partial x}, L_{2}\right\rangle-i \partial_{x}^{-1}\left\langle\left[L_{1}-L_{-1}, K_{0 ; \alpha}^{ \pm}(x, \lambda)\right], L_{2}\right\rangle \\
& \left\langle K_{1 ; \alpha}^{ \pm}, L_{1}\right\rangle=k_{01, \alpha}^{ \pm}-\partial_{x}^{-1}\left\langle\frac{\partial K_{1 ; \alpha}^{ \pm, \perp}}{\partial x}, L_{1}\right\rangle,
\end{aligned}
$$


where $k_{01}^{ \pm}$and $h_{01}^{ \pm}$are matrix-valued integration constants.

Skipping the details we obtain:

$$
\begin{aligned}
\Lambda_{1} K_{1 ; \alpha}^{ \pm, \perp} & =\left(\lambda+\lambda^{-1}\right) H_{1 ; \alpha}^{ \pm, \perp}-\operatorname{ad}_{L_{1}}^{-1}\left(\left[L_{1}-L_{-1}, h_{00}\right]+\frac{3}{2} L_{2, x} \partial_{x}^{-1}\left\langle\left[L_{2}, L_{-1}\right], k_{00}\right\rangle\right) \\
\Lambda_{2} H_{1 ; \alpha}^{ \pm, \perp} & =\left(\lambda+\lambda^{-1}\right) K_{1 ; \alpha}^{ \pm, \perp}+\frac{3 i}{2} \operatorname{ad}_{L_{1}}^{-1}\left(L_{2, x} h_{01}+i L_{2, x} \partial_{x}^{-1}\left\langle\left[L_{2}, L_{-1}\right], k_{00}\right\rangle\right) \\
& -\operatorname{ad}_{L_{1}}^{-1}\left(\left[L_{1}-L_{-1}, k_{00}\right]\right)
\end{aligned}
$$

where $h_{00}$ and $k_{00}$ are arbitrary constants which we shall set to be equal to zero. The operators $\Lambda_{j}, j=1,2$ are defined as follows:

$$
\begin{aligned}
\Lambda_{1} K_{1 ; \alpha}^{ \pm, \perp} & =-i \operatorname{ad}_{L_{1}}^{-1}\left(\frac{\partial K_{1 ; \alpha}^{ \pm, \perp}}{\partial x}-\left(\mathbb{1}-\varphi_{0}\right)\left[L_{1}-L_{-1}, \partial_{x}^{-1}\left[L_{1}, K_{1 ; \alpha}^{ \pm, \perp}\right]\right]\right) \\
+ & \frac{i}{2} \operatorname{ad}_{L_{1}}^{-1} L_{1, x} \partial_{x}^{-1}\left\langle\frac{\partial K_{1 ; \alpha}^{ \pm, \perp}}{\partial x}, L_{1}\right\rangle \\
\Lambda_{2} H_{1 ; \alpha}^{ \pm, \perp} & =-\operatorname{iad}_{L_{1}}^{-1}\left(\frac{\partial H_{1 ; \alpha}^{ \pm, \perp}}{\partial x}-\left(\mathbb{1}-\varphi_{0}\right)\left[L_{1}-L_{-1}, \partial_{x}^{-1}\left[L_{1}, H_{1 ; \alpha}^{ \pm, \perp}\right]\right]\right. \\
& \left.-\frac{3}{2} L_{2, x} \partial_{x}^{-1}\left(\left\langle\frac{\partial H_{1 ; \alpha}^{ \pm, \perp}}{\partial x}, L_{2}\right\rangle+\left\langle\left[L_{2}-L_{-2}, L_{1}-L_{-1}\right], \partial_{x}^{-1}\left[L_{1}, H_{1 ; \alpha}^{ \pm, \perp}\right]\right\rangle\right)\right)
\end{aligned}
$$

Thus, we obtain:

$$
\begin{aligned}
& \Lambda_{2} \Lambda_{1} K_{1 ; \alpha}^{ \pm, \perp}=\left(\lambda+\lambda^{-1}\right)^{2} K_{1 ; \alpha}^{ \pm, \perp} \\
& \Lambda_{1} \Lambda_{2} H_{1 ; \alpha}^{ \pm, \perp}=\left(\lambda+\lambda^{-1}\right)^{2} H_{1 ; \alpha}^{ \pm, \perp}
\end{aligned}
$$

\section{Conclusions}

We derived the recursion operators $\Lambda$ for the system (1.4) related to the symmetric space $S U(3) / S(U(1) \times U(2))$ with an additional $\mathbb{Z}_{2}$-reduction. Our first derivation of $\Lambda$ is based on the GKS method [10].

Another way to construct $\Lambda$ consists in using the Wronskian relations. They allow us to determine the 'squared solutions' of $L$ which are treated as eigenfunctions of $\Lambda$.

Our results could be extended for operators $L$ related to a generic symmetric space of the type A.III $\cong S U(n+k) / S(U(n) \times U(k))$ as well as to other types of symmetric spaces.

\section{Acknowledgements}

The authors acknowledge support from the Royal Society and the Bulgarian academy of sciences via joint research project "Reductions of Nonlinear Evolution Equations and analytic spectral theory". V.S.G. and G.G.G. are thankful to the organizers of the international workshop: "Nonlinear Physics: Theory and Experiment. VI" for the financial support and the warm hospitality in Gallipoli. The work of G.G.G. is supported by the Science Foundation of Ireland (SFI), under Grant No. 09/RFP/MTH2144. 


\section{A Some intermediate results}

In this appendix we will present some intermediate results, used in section 4 . The involutions $J$ induce a $\mathbb{Z}_{2}$ grading in the Lie algebra $\mathfrak{g}$ as follows:

$$
\begin{array}{rlrl}
\mathfrak{g} & \equiv \mathfrak{g}^{(0)} \oplus \mathfrak{g}^{(1)}, & & \\
\mathfrak{g}^{(0)} & \equiv\left\{Y: Y=J Y J^{-1}\right\}, & \mathfrak{g}^{(1)} \equiv\left\{X: X=-J X J^{-1}\right\} \\
Y & =\left(\begin{array}{ccc}
-k-n & 0 & 0 \\
0 & k & m \\
0 & m^{*} & n
\end{array}\right), & X & =\left(\begin{array}{ccc}
0 & a & b \\
a^{*} & 0 & 0 \\
b^{*} & 0 & 0
\end{array}\right)
\end{array}
$$

The inner automorphism $\varphi_{0}$ staying in the formula for the "extended squared solutions" (4.9) can be represented in the form:

$$
\begin{aligned}
\varphi_{0}(Z) \equiv K_{0} Z K_{0}^{-1}, & Y^{+}=\left(\begin{array}{ccc}
-k-n & 0 & 0 \\
0 & k & 0 \\
0 & 0 & n
\end{array}\right), \quad Y^{-}=\left(\begin{array}{ccc}
0 & 0 & 0 \\
0 & 0 & m \\
0 & m^{*} & 0
\end{array}\right), \\
X=X^{+}+X^{-}, \quad X^{+}=\left(\begin{array}{ccc}
0 & 0 & b \\
0 & 0 & 0 \\
b^{*} & 0 & 0
\end{array}\right), & X^{-}=\left(\begin{array}{ccc}
0 & a & 0 \\
a^{*} & 0 & 0 \\
0 & 0 & 0
\end{array}\right),
\end{aligned}
$$

Next we take into account that $\varphi_{0}\left(H_{0}\right)=H_{0}$ and $\varphi_{0}\left(K_{0}\right)=K_{0}$ and therefore:

$$
\begin{aligned}
& \left\langle\left[L_{1}-L_{-1}, K_{0 ; \alpha}^{ \pm}(x, \lambda)\right], L_{2}\right\rangle=2|u|^{2}\left(\kappa_{0, \alpha}^{ \pm} v^{*}-\kappa_{0, \alpha}^{ \pm, *} v\right) \\
& \left\langle\left[L_{1}-L_{-1}, H_{0 ; \alpha}^{ \pm}(x, \lambda)\right], L_{1}\right\rangle=0
\end{aligned}
$$

where

$$
K_{0, \alpha}^{ \pm}=\left(\begin{array}{ccc}
0 & 0 & \kappa_{0, \alpha}^{ \pm} \\
0 & 0 & 0 \\
\kappa_{0, \alpha}^{ \pm, *} & 0 & 0
\end{array}\right), \quad H_{0, \alpha}^{ \pm}=\left(\begin{array}{ccc}
-k_{0, \alpha}^{ \pm}-n_{0, \alpha}^{ \pm} & 0 & 0 \\
0 & k_{0, \alpha}^{ \pm} & 0 \\
0 & 0 & n_{0, \alpha}^{ \pm}
\end{array}\right)
$$

Introduce the notations

$$
K_{1, \alpha}^{ \pm}=\left(\begin{array}{ccc}
0 & w_{1, \alpha}^{ \pm} & z_{1, \alpha}^{ \pm} \\
w_{1, \alpha}^{ \pm, *} & 0 & 0 \\
z_{1, \alpha}^{ \pm, *} & 0 & 0
\end{array}\right), \quad H_{1, \alpha}^{ \pm}=\left(\begin{array}{ccc}
-k_{1, \alpha}^{ \pm}-n_{1, \alpha}^{ \pm} & 0 & 0 \\
0 & k_{1, \alpha}^{ \pm} & m_{1, \alpha}^{ \pm} \\
0 & m_{1, \alpha}^{ \pm \pm,} & n_{1, \alpha}^{ \pm}
\end{array}\right)
$$

Then

$$
\begin{aligned}
\left\langle H_{1 ; \alpha}^{ \pm}, L_{2}\right\rangle & =h_{01, \alpha}^{ \pm}-\partial_{x}^{-1}\left\langle\frac{\partial H_{1 ; \alpha}^{ \pm, \perp}}{\partial x}, L_{2}\right\rangle-2 i \partial_{x}^{-1}|u|^{2}\left(v^{*} k_{00, \alpha}^{ \pm}-v k_{00, \alpha}^{ \pm, *}\right) \\
& +8 \partial_{x}^{-1}|u|^{2}\left(v^{*} \partial_{x}^{-1}\left(u m_{1, \alpha}^{ \pm}\right)+v \partial_{x}^{-1}\left(u^{*} m_{1, \alpha}^{ \pm, *}\right)\right) \\
\left\langle K_{1 ; \alpha}^{ \pm}, L_{1}\right\rangle & =k_{01, \alpha}^{ \pm}-\partial_{x}^{-1}\left\langle\frac{\partial K_{1 ; \alpha}^{ \pm, \perp}}{\partial x}, L_{1}\right\rangle
\end{aligned}
$$




\section{References}

[1] Ablowitz M J, Kaup D J, Newell A C, Segur H., The inverse scattering transform - Fourier analysis for nonlinear problems, Studies in Appl. Math. 53 (1974), n 4, 249-315.

[2] V.E. Adler, A.B. Shabat, and R.I. Yamilov, The symmetry approach to the problem of integrability, Theor. Math. Phys. 125(2000), No.3, 1603-1661.

[3] Drinfel'd V and Sokolov V V 1985 Lie Algebras and Equations of Korteweg -de Vries Type Sov. J. Math. 30, 1975-2036.

[4] V. S. Gerdjikov, E. Kh. Khristov. On the evolution equations solvable with the inverse scattering problem. II. Hamiltonian structures and Backlund transformations. Bulgarian J. Phys. 7, No.2, 119-133, (1980) (In Russian).

[5] V. S. Gerdjikov and M. I. Ivanov The quadratic bundle of general form and the nonlinear evolution equations. II. Hierarchies of Hamiltonian structures. Bulg. Jour. Phys., 10, 130-143 (1983) (In Russian).

[6] Gerdjikov V., Mikhailov A. and Valchev T. Reductions of Integrable Equations on A.III-type Symmetric Spaces, Jour. Phys. A: Math Theor. 43 (2010) 434015.

[7] V. Gerdjikov, A. Mikhailov, T. Valchev. Recursion Operators and Reductions of Integrable Equations on Symmetric Spaces. J. Geometry and Symmetry in Physics 20, 1-34 (2010).

[8] Gerdjikov V S, Vilasi G and Yanovski A B 2008 Integrable Hamiltonian Hierarchies. Spectral and Geometric Methods Lecture Notes in Physics 748 (Springer Verlag, Berlin, Heidelberg, New York).

[9] Golubchik I Z and Sokolov V V 2000 Multicomponent Generalization of the Hierarchy of the Landau-Lifshitz Equation Theor. and Math. Phys. 124 n. 1 909-917.

[10] M. Gürses, A. Karasu and V. Sokolov, On Construction of Recursion Operators From Lax Representation, J. Math. Phys. 40 (1999), no.12, 6473-6490 (Arxiv: 9909003v1 [solv-int]).

[11] Helgasson S 1978 Differential Geometry, Lie Groups and Symmetric Spaces (Academic Press, New York).

[12] D. J. Kaup, A. C. Newell. An exact solution for a derivative nonlinear Schrodinger equation J. Math. Phys. 19, 798 (1978); doi:10.1063/1.523737,

[13] Lombardo S and Sanders J, On the Classification of Automorphic Lie Algebras, Commun. Math. Phys. 299 (2010), 793-824 (arXiv:0912.1697v1 [nlin.SI]).

[14] Mikhailov A V 1979 On the Integrability of Two-dimensional Generalization of the Toda Lattice Lett. in Jour. of Exper. and Theor. Phys. 30 443-448.

[15] Mikhailov A V 1980 Reductions in Integrable Systems. The Reduction Groups Lett. in Jour. of Exper. and Theor. Phys. 32 187-192. 
[16] Mikhailov A 1981 The Reduction Problem and The Inverse Scattering Method Physica $D$ 3 73-117.

[17] Mikhailov A V 1982 The Landau-Lifschitz Equation and the Riemman Boundary Problem on a Torus, Phys. Lettrs. A 9251.

[18] Mikhailov A and Lombardo S 2004 Reductions of Integrable Equations: Dihedral Group Jour. Phys. A 37 7727-7742.

[19] Mikhailov A and Lombardo S 2005 Reduction groups and Automorphic Lie Algebras. Commun. Math. Phys., 258, pp. 179-202.

[20] Mikhailov A V, Olshanetski M A and Perelomov A M 1981 Two-dimensional Generalized Toda Lattice Commun. Math. Phys. 79 473-488.

[21] A.V. Mikhailov, V.V. Sokolov, Symmetries of Differential Equations and the Problem of Integrability, In: Integrability, Ed: A. V. Mikhailov, Lect. Notes in Physics 767, Springer Verlag (2009), pp. 19-88.

[22] Takhtadjan L. and Faddeev L., The Hamiltonian Approach to Soliton Theory, Springer Verlag, Berlin, 1987.

[23] Wang J. P. Lenard Scheme for Two-dimensional Periodic Volterra Chain, Jour. Math. Phys. 50 (2009) 023506.

[24] Zakharov V., Manakov S., Novikov S. and Pitaevskii L., Theory of Solitons: The Inverse Scattering Method, Plenum, New York, 1984. 